\title{
Multiple Interneural Communications of Brachial Plexus-Anatomical Description and Clinical Significance
}

\author{
Jasbir K, Shivi G (凶), Vandana M, Rajesh KS, Gayatri R \\ Department of Anatomy, Vardhman Mahavir Medical College and Safdarjung Hospital, New Delhi- \\ 110029, India.
}

\begin{abstract}
Brachial plexus blockade requires efficient and thorough understanding of the detailed anatomy of the plexus and the variations associated with the plexus. Anomalies associated with the branches of the plexus may complicate attempts of nerve blockade for anesthesia. The present study reports a rare neural communication between ulnar and radial nerves, observed in the left axilla at high humeral level, $6 \mathrm{~cm}$ distal to tip of coracoid process of scapula and $2.8 \mathrm{~cm}$ proximal to the entry of the radial nerve into the radial groove. The communicating ramus also gave a branch to the medial head of triceps brachii. Musculocutaneous and median nerve connection was also observed in the arm, $9 \mathrm{~cm}$ distal to the tip of coracoid process. The studies regarding the communication between ulnar and radial nerve are rare and the coexistence of this variation along with median and musculocutaneous nerve communication is unique. Knowledge of such rare variations and anomalies are important to minimize the possible complications of regional anesthesia and surgery. The present report is a humble attempt to reinforce awareness of such neural variations among clinicians.
\end{abstract}

Keywords: $\quad$ brachial plexus, median, musculocutaneos, nerve, radial, ulnar

\section{Correspondence:}

Shivi Goel, Department of Anatomy, Vardhman Mahavir Medical College and Safdarjung Hospital, New Delhi-110029, India. Tel: +91 9717186546 Fax: +91 1123011617 Email: sm.8sep@gmail.com.

\section{Introduction}

Detailed understanding of brachial plexus and its anatomic variations is extremely important for effective nerve blockade in this region. The present study depicts a unique case of radio ulnar communication in the left axilla. Many authors have reported communications between the branches of brachial plexus $(1,2)$. However, literature regarding communication between the branches of medial and posterior cords of brachial plexus is rare, thus quantifying a need for further research of such variations and anomalies.

The study aims to report this unusual variation so as to assist the anesthetists in their attempt to block the nerves and orthopaedicians in assessing sensorimotor symptoms while diagnosing nerve injuries.

\section{Case Report}

The present variation of brachial plexus was incidentally observed during routine dissection of the left upper limb of a 50-year-old female cadaver. A rare communication between radial and ulnar nerves was observed in the left axilla at high humeral level, $6 \mathrm{~cm}$ distal to tip of coracoid process of scapula (Fig. 1). This communicating twig (* in Fig 1) commenced from the radial nerve, $2.8 \mathrm{~cm}$ proximal to its entry into the spiral groove. The communicating ramus joined the ulnar nerve $3 \mathrm{~cm}$ proximal to the point where the latter pierced medial intermuscular septum. This communication, measuring $6.5 \mathrm{~cm}$ in length, was placed posteromedial to distal part of axillary artery and proximal part of brachial artery. It also gave a branch to medial head of triceps brachii (\# in Fig 1); the latter was given $0.3 \mathrm{~cm}$ 
distal to the origin of communication and measured $4 \mathrm{~cm}$ in length. The medial head of triceps was also supplied by a branch, given directly by radial nerve in the axilla. Additionally, the dissection also displayed a communication between musculocutaneous nerve $(\mathrm{MCN})$ and median nerve $(\mathrm{MN})$ in the left arm, 9cm distal to the tip of coracoid process of scapula (@ in Fig. 1). This communicating branch, $3.5 \mathrm{~cm}$ in length, was given off from MCN after the latter pierced the coracobrachialis muscle. It joined the $\mathrm{MN} 13 \mathrm{~cm}$ distal to the formation of the nerve by union of medial and lateral roots. The other branches of brachial plexus and adjoining vessels including axillary artery and vein displayed usual anatomy. No unusual anatomical variation was detected in the right arm.

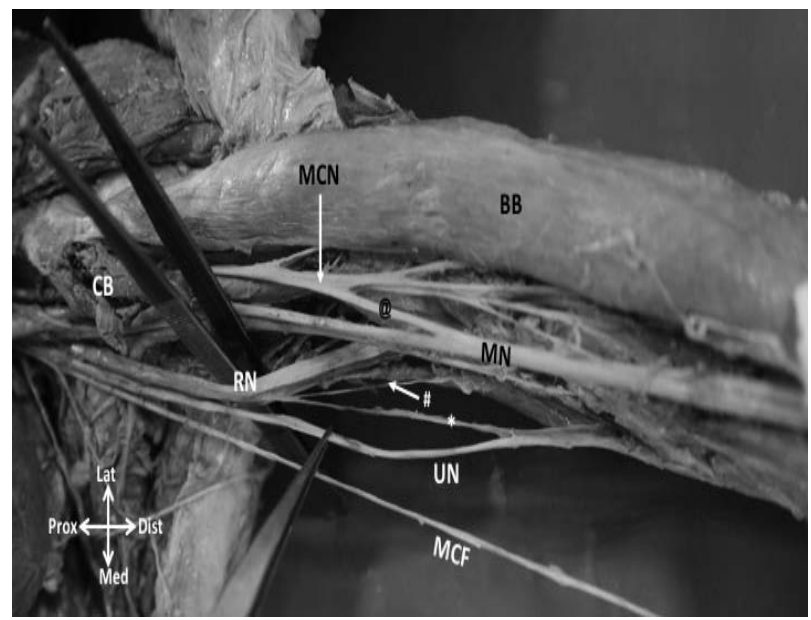

Figure 1: Left arm (anteromedial view) showing: $\mathrm{CB}$ coracobrachialis muscle; $\mathrm{BB}$ - biceps brachii muscle; $\mathrm{MN}$ median nerve; $\mathrm{MCN}$ - musculocutaneous nerve; $\mathrm{RN}$ - radial nerve; UN - ulnar nerve; MCF - medial cutaneous nerve of forearm; @ - communication between MCN and MN; * communication between $\mathrm{RN}$ and $\mathrm{UN}$; \# - branch from * to triceps brachii; Lat - lateral; Med - medial; Prox - proximal; Dist - distal

\section{Discussion}

Various communications between different branches of brachial plexus and its cords have been reported in previous studies $(1,2)$. However, communication between radial nerve and ulnar nerve is a rare finding. Literature suggests that the branches of brachial plexus develop in two planes - anterior and posterior. The nerves of the medial and lateral cords belong to the anterior plane and nerves of posterior cord belong to the posterior plane. Communications and variations are most frequently observed between the nerves of the same plane, thus explaining the common occurrence of communications between lateral and medial cords and their branches and rare occurrence of radioulnar communications (1). The uniqueness of the present investigation lies in the fact that the radioulnar communion encountered in the arm also provided a twig to the medial head of triceps brachii. To the best of our knowledge, this finding has not been reported earlier. The medial head of triceps thus is observed to receive nerve supply from three different sources, from the radial nerve in axilla and in spiral groove, and another branch from the communication between radial and ulnar nerve in the arm. In view of its innervation, the medial head of triceps brachii can justifiably be designated as a composite muscle. The anatomical scenario of present report also suggests that in the event of injury to radial nerve, the medial head of triceps brachii would be spared. Moreover, the authors strive to report this unusual neural variation in an attempt to provide anatomists a base for further studies in this direction.

The dissection also displayed a communication between MCN and MN. The musculocutaneous - median nerve communication has been classified into different types. Type I being where MCN communicates with MN before piercing coracobrachialis muscle, Type II where MCN communicates with MN after piercing coracobrachialis and Type III where MCN does not pierce coracobrachialis (1). According to this classification, our study displays Type II communication between MCN and MN.

Such variations can be described on the basis of embryological development of nerves of brachial plexus. Site specific expression of some chemotactic factors govern the path taken up by developing axons. Any deviation in such signalling pathways which regulate the neuronal growth cones and mesenchymal cells can result in significant variations. The developmental differences once formed would persist postnataly $(3,4,5,6)$. Thus, the lack of coordination between the formation of the limb muscles and their innervation may result in the appearance of aberrant communications between different cords and their branches.

Further some investigators have considered the theory of ontogeny recapitulating phylogeny for the interpretation of the nerve anomalies of the arm (7). Studies report the presence of such communications in monkeys and apes. Their occurrence in humans depicts the primitive motor supply of muscles of upper limb (3).

Anatomic variations of peripheral nerves are important for Orthopedic Surgeons, Neurophysicians, Physiotherapists and Radiologists. Awareness of such unusual neural variations as noted in the present study may prove valuable in diagnosing peripheral 
neuropathies and in nerve grafting. Further their knowledge is of immense importance in planning radical neck dissections, managing shoulder joint trauma and in treatment of fracture of surgical neck of humerus. Also, their proper understanding helps orthopedic surgeons in assessing sensorimotor symptoms while diagnosing nerve injuries. It has been stated that anomalous origin, course and distribution of the various branches of brachial plexus and its cords may succumb to iatrogenic injuries and entrapment neuropathies (8). Such neural variations if ignored, may pose problems for the radiologists and anesthetists leading to ineffective nerve blockade. Moreover, the treatment of complex regional pain syndrome can be complicated due to unusual communications between the branches of brachial plexus because they carry the sympathetic supply to the upper limb.

In summary, the knowledge of anatomical aberrations in the brachial plexus are extremely important for diagnosing peripheral neuropathies, nerve grafting and for effective peripheral nerve blockade in this region. This report is a humble attempt to delineate the potential clinical implications and possible embryological explanation of the said variations along with a brief description of their phylogenetic basis.

\section{References}

1. Venieratos D, Anagnostopoulou S. Classification of communications between the musculocutaneous and median nerves. Clin Anat 1998; 11(5): 327-31.
2. Goel S, Rustagi SM, Kumar A, Mehta V, Suri RK. Multiple unilateral variations in medial and lateral cords of brachial plexus and their branches. Anat Cell Biol 2014; 47(1): 77-80.

3. Miller RA. Comparative studies upon the morphology and distribution of the brachial plexus. Am J Anat 1934; 54(1): 143-75.

4. Larsen WJ. Development of the limbs. In: Human embryology. New York: Churchill Livingstone, 1993, pp-290-1.

5. Brown MC, Hopkins WG, Keynes RJ. Essentials of neural development - Axon guidance and target recognition. Cambridge: Cambridge University Press, 1991, pp-46-66.

6. Sannes HD, Reh TA, Harris WA. Development of nervous system - Axon growth and guidance. New York: Academic Press, 2000, pp-189-97.

7. Chauhan R, Roy TS. Communication between the median and the musculocutaneous nerves: A case report. J Anat Soc India 2002; 51(1): 72-5.

8. Roberts WH. Anomalous course of the median nerve medial to the trochlea and anterior to the medial epicondyle of the humerus. Ann Anat 1992; 174(4): 309-11. 